www.jmscr.igmpublication.org

Index Copernicus Value: 79.54

ISSN (e)-2347-176x ISSN (p) 2455-0450

crossrefDOI: https://dx.doi.org/10.18535/jmscr/v7i1.132

\title{
Treatment of Radicular Cyst with Platelet Rich Fibrin after Enucleation: A Case Report
}

\author{
Authors \\ Dr Arka Das ${ }^{1^{*}}$, Dr Sunita R. Pal ${ }^{2}$ \\ ${ }^{1}$ Assistant Professor, M B KEDIA Dental College, Birgunj, NEPAL \\ ${ }^{2}$ Post graduate, Department of Periodontology, Rajarajeswari Dental College and Hospital, \\ Bangalore-560074 \\ *Corresponding Author \\ Dr Arka Das \\ Dept of Oral and Maxillofacial Surgery, MB Kedia Dental College, Birgunj, Nepal \\ Email:dr.arkadas@outlook.com, Tel: +917829483141,+9779826251195
}

\section{Introduction}

The radicular cyst (periapical cyst) is the common cyst of odontogenic origin. Most commonly the cause of radicular cyst is an infected or a non-vital tooth with pulpal necrosis. The toxins leave the apical end of the tooth which leads to periapical inflammation. The periapical inflammation in turn stimulates the rest of malassez present in the apical periodontal ligament leading to a formation of periapical granuloma. In due course this epithelium undergoes necrosis due to lack of blood supply and the granuloma transforms into a cyst (periapical cyst). Usually the lesions when small are clinically asymptomatic and are discovered as clinical or radiologic findings during regular dental check up.

Platelet rich fibrin as described by choukron et al ${ }^{1}$ is a second generation platelet concentrate which contains platelets and growth factors in the form of fibrin membranes prepared from the patient's own blood free of any anticoagulant or other artificial biochemical modifications ${ }^{1,2}$. Autologous PRF is said to have healing capabilities as a biomaterial and presently studies have been conducted which show its application in various disciplines of dentistry and oral and maxillofacial surgery.

After activation of platelets which are trapped in the fibrin matrix, growth factors are released and stimulate the mitogenic response in the bone periosteum during normal wound healing for repair of the bone ${ }^{3}$

PRF represent a new revolutionary step in platelet gel therapeutic concept ${ }^{4}$. The platelets and leukocyte cytokines play an important role in the this biomaterial, and the fibrin matrix supporting this is very helpful in determining the element responsible for the healing ${ }^{5}$

\section{Case Report}

A 38 year old female patient walked into the department of oral and maxillofacial surgery of MB KEDIA DENTAL COLLEGE, Nepal with a complaint of slighltly mobile right maxillary lateral incisor. Patient had no other associated symptoms. Patient gave a history of minor trauma 
to upper front tooth around 15 months back. On general dental examination the maxillary right lateral incisor (tooth no. 12) was found to be grade 1 mobile and the maxillary right canine was tilted mesiodistally. When enquired about the canine inclination patient answered that the inclination of the canine has started about a 15 months ago. Patient was advised an Intra oral periapical radiograph of 12,13 region and an orthopantogram. On the radiograph there was a well definedperiapical radiolucecy present with respect to the right maxillary lateral incisor and the radiolucency was also involving the right maxillary lateral incisor (11). The radiolucency measured approximately $1.5 \mathrm{~cm}$ mesidistally and 1 $\mathrm{cm}$ superioinferiorly. The roots of the canine (13) were present distal to the radiolucency indicating that it had pushed the canine due to the pressure exerted. On electric pulp testing the lateral incisor gave no response and the central incisor gave delayed response indicating that the pulp of the lateral incisor was non vital. Enucleation of the lesion was planned under local anesthesia after endodontic treatment of the maxillary right central and lateral incisor. During the course of the treatment the lateral incisor was found to have higher chances of endodontic failure that's why endodontic treatment was completed only in the central incisor.

After the endodontic treatment the patient was planned to go ahead with enucleation. Under proper aseptic conditions and optimal anesthesia crevicular insions were placed from the right $2^{\text {nd }}$ maxillary premolar to the left lateral incisor (15 to 22). Once the incision was placed a full thicknes mucoperiosteal flap was elevated. The moment the flap was elevated cystic fluid started to ooze out and after a little bit of elevation it was seen that the cyst had eroded the buccal aspect of the maxillary bone $1 \mathrm{~cm} \times 1 \mathrm{~cm}$ superior inferiorly and mesiodistally. The cystic fluid was drained and the depth was assessed. The depth was found to be around $5 \mathrm{~cm}$ extending anteroposteriorly and $7 \mathrm{~cm}$ mesiodistally which was not evident on the radiograph. The cystic lining of the lesion was removed fully. The diseased bone was also removed using bone rongeur and HP-6 round bur. The right maxillary lateral incisor tooth was extracted. It was found that the teeth had a horizontal fracture at the apical third because of which the pulp had become necrotic and caused the cyst to form. Due to the size of the lesion it was decided to use platelet rich fibrin and freeze dried bone graft in the lesion to accelerate bone formation during healing process. Due to the patients poor socioeconomic conditions the patient did not consent to the placement of the bone graft so it was decided to go ahead with the placement of platelet rich fibrin and close the defect.

\section{Preparation of PRF}

The protocol for PRF preparation is very simple and simulates that of PRP. Whole venous blood is collected in the two sterile vacutainer tubes $(6 \mathrm{ml})$ without anticoagulant and the vacutainer tubes are then placed in a centrifuge machine at 3,000 revolutions per minute (rpm) for $10 \mathrm{~min}$, after which it settles into three layers: Upper strawcolored acellular plasma, middle fraction containing the fibrin clot, and red-colored lower fraction containing red blood cells (RBCs). The upper straw-colored layer is then removed and middle fraction is collected which is the PRF. A fibrin clot is then formed in the middle between the red corpuscles at bottom and acellular plasma at the top. The middle part is numerous platelets entrapped in fibrin meshes. The success of this technique entirely depends on time gap between the blood collection and its transfer to the centrifuge and it should be done immediately. The blood sample without anticoagulant, starts to coagulate almost immediately upon contact with the glass, and it decreases the time of centrifugation to concentrate fibrinogen. Following proper protocol and quick handling is the only way to obtain a clinically usable PRF clot charged with serum and platelets.

Once the platelet rich fibrin was developed it was placed in the defect and the incision was closed using 3-0 vicryl. Patient was put on antibiotics and 


\section{JMSCR Vol||07||Issue||01||Page 768-771||January}

anti-inflammatory medication and recalled for follow up.

At 2 months follow up patient is pain free and and has no complaints. The operated site has healed intraorally. An orthopantogram and a maxillary occlusal view was done.

Notes: Ideally CBCT / CT should have been done pre-operatively To assess the extent of the lesion. Also the use of bone graft materials would have been beneficial to the patient. But these were not available looking at the patients poor socioeconomic status and the also the region where the patient presented.

\section{Preoperative Radiographs}
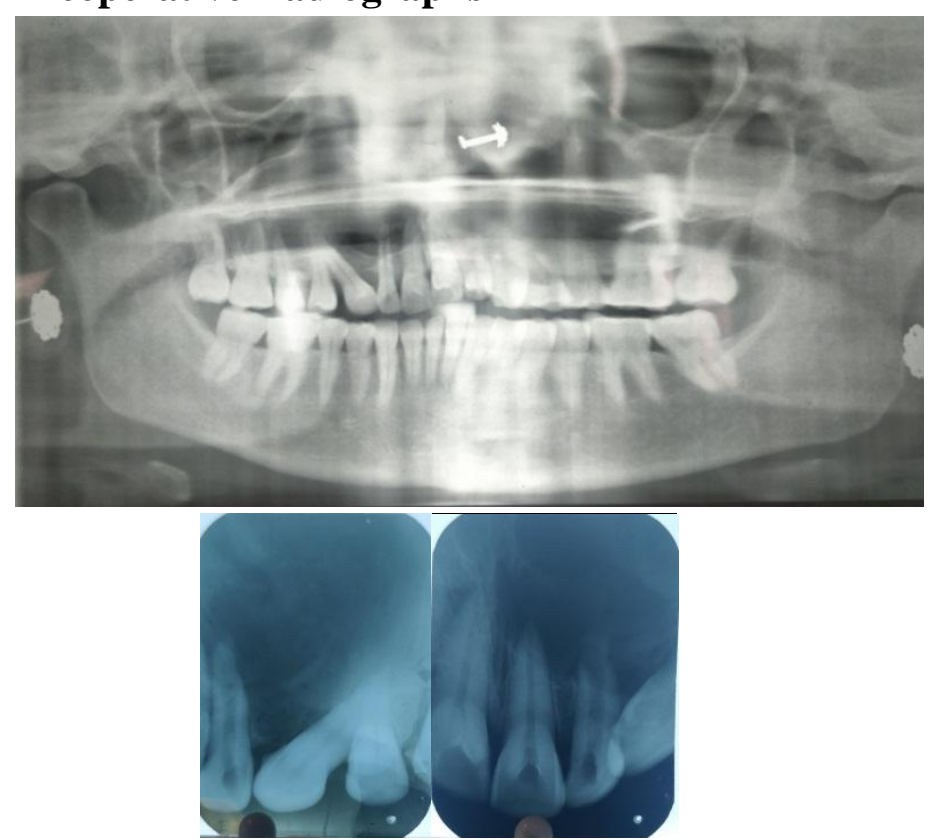

Figure $\mathbf{1} \& \mathbf{2}$ Orthopantogram and IOPAR showing radiolucency wrt 12 region and canine tilted mesiodistally

\section{Intra Operative Photographs}

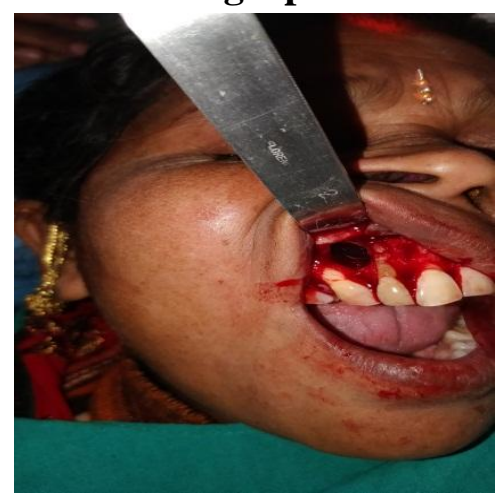

Figure $\mathbf{3}$ immediately after elevation of mucoperiosteal flap

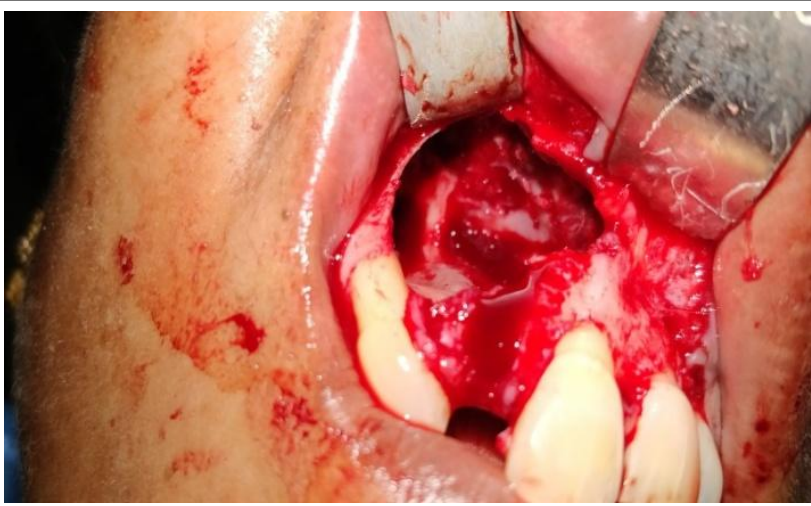

Figure 4: after removal of cystic lining and diseased bone

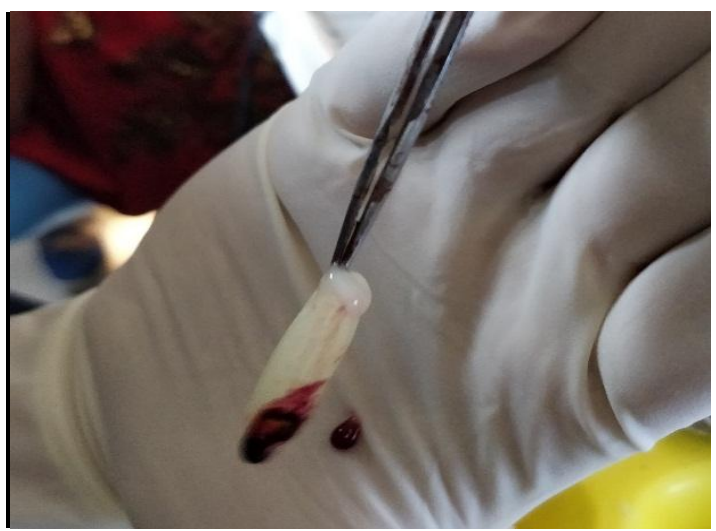

Figure 5 : Platelet Rich Fibrin

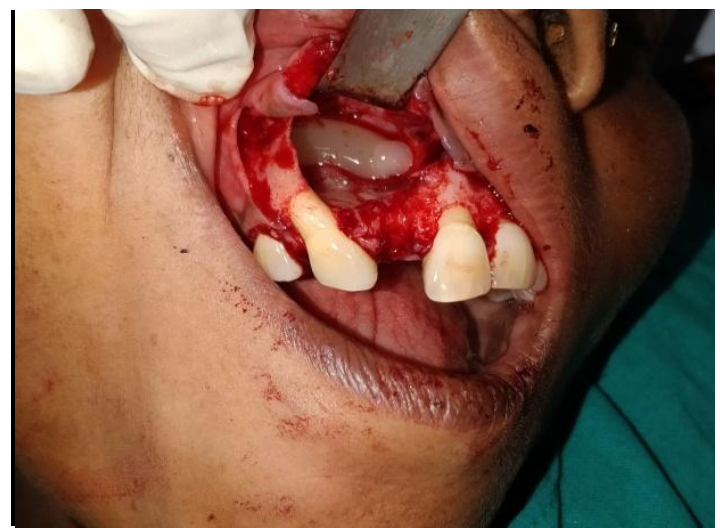

Figure 6: PRF placed in the cystic space for accelerated bone healing

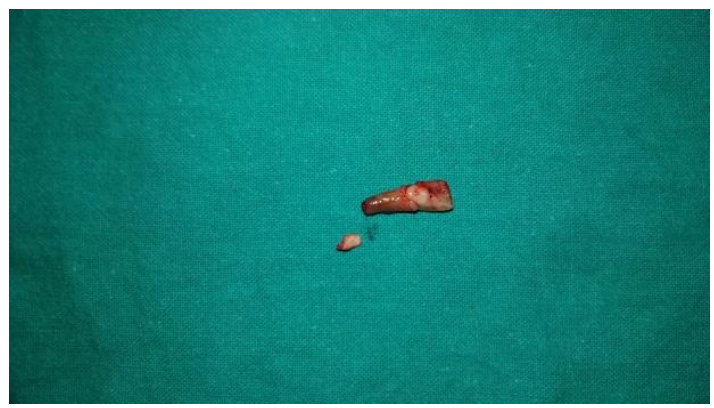

Figure 7: the extracted tooth with a fracture in the apical third of the root 


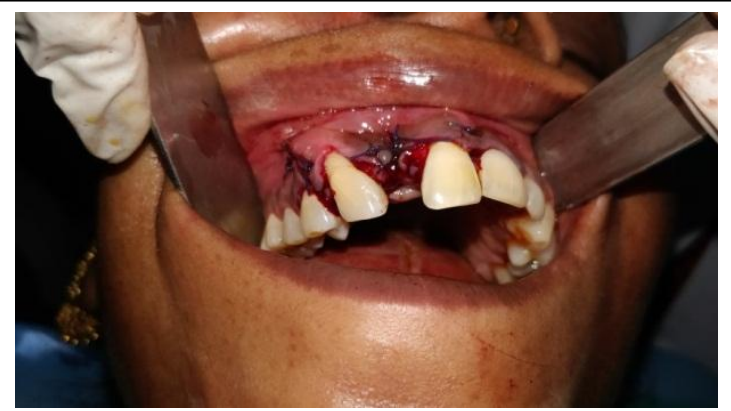

Figure 8: sutures placed using vicryl 3-0 sutures

\section{Post Operative Radiograph (2 Months)}

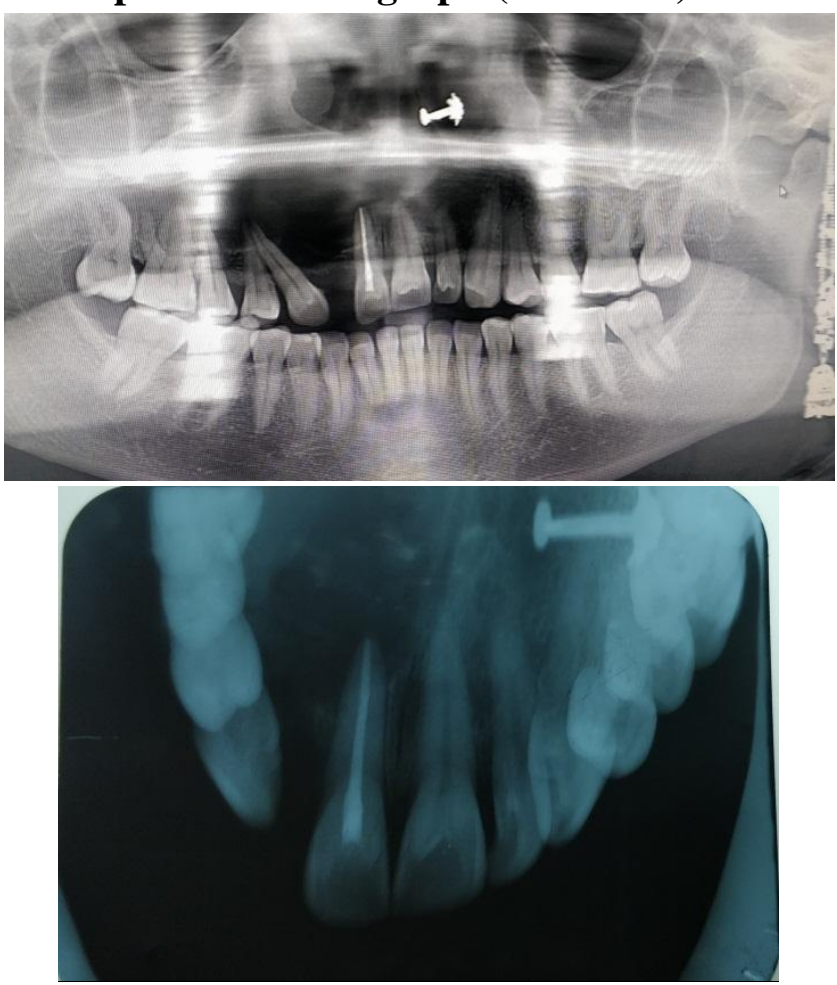

Figure 8 \& 9: 2 month post operative OPG and maxillary occlussal radiograph

\section{References}

1. Dohan DM, Choukroun J, Diss A, Dohan SL, Dohan AJ, et al. (2006) Platelet-rich fibrin (PRF): a second-generation platelet concentrate, part I: technological concepts and evolution. Oral Surg Oral Med Oral Pathol Oral RadiolEndod 101: e37-e44.

2. Gupta V, Bains BK, Singh GP, Mathur A, Bains R (2011) Regenerative potential of platelet rich fibrin in dentistry: Literature review. Asian J Oral Health Allied Sci 1: 22-28.

3. Gassling V, Douglas T, Warnke PH, Açil Y, Wiltfang J, Becker ST. Platelet-rich fibrin membranes as scaffolds for periosteal tissue engineering. Clin Oral Implants Res. 2010;21:543-9

4. Dohan DM, Choukroun J, Diss A, Dohan SL, Dohan AJ, Mouhyi J, et al. Plateletrich fibrin (PRF): A second-generation platelet concentrate. Part I: Technological concepts and evolution. Oral Surg Oral Med Oral Pathol Oral Radiol Endod. 2006;101:e37-44.

5. Balaram Naik, P Karunakar, ${ }^{1}$ M Jayadev, ${ }^{1}$ and V Rahul Marshal ${ }^{2}$ Role of Platelet rich fibrin in wound healing: A critical review $\mathbf{J}$ Conserv Dent. 2013 Jul-Aug; 16(4): 284293.doi: 10.4103/0972-0707.114344. 